\title{
Resistive Switching in Aqueous Nanopores by
}

\section{Shock Electrodeposition}

Ji-Hyung Han ${ }^{1,2, a}$, Ramachandran Muralidhar ${ }^{3}$, Rainer Waser ${ }^{4,5,6, b}$ and Martin Z. Bazant ${ }^{1,2, b, c^{*}}$

\begin{abstract}
Solid-state programmable metallization cells have attracted considerable attention as memristive elements for Redox-based Resistive Random Access Memory (ReRAM) for low-power and low-voltage applications. In principle, liquid-state metallization cells could offer the same advantages for aqueous systems, such as biomedical lab-on-a-chip devices, but robust resistive switching has not yet been achieved in liquid electrolytes, where electrodeposition is notoriously unstable to the formation of fractal dendrites. Here, the recently discovered physics of shock electrodeposition are harnessed to stabilize aqueous copper growth in polycarbonate nanopores, whose surfaces are modified with charged polymers. Stable bipolar resistive switching is demonstrated for 500 cycles with $<10$ s retention times, prior to any optimization of the geometry or materials.
\end{abstract}




\section{Introduction}

In recent years, memristive elements[1] based on internal redox-reactions have attracted intense interest for applications such as non-volatile Random Access Memory[2-4]. Resistive switching in solid-state ultra-thin films is widely viewed as a potential replacement for flash memory in devices requiring lower power, lower voltage, and higher programming speed. A popular class of these Redox-based Resistive Random Access Memories (ReRAM) is based on the reversible dielectric breakdown of transition metal oxide (TMO) films, in which oxygen vacancies (or transition metal interstitials) are moved and the valence change of the metal cations leads to the formation of conducting filaments[4,5]. This class is called Valence Change Memories (VCM), also known as OxRRAM. Another important class relies on an electrochemically active electrode material such as $\mathrm{Ag}$ or $\mathrm{Cu}$ and ultra-thin solid electrolyte or insulator films. This class is called electrochemical metallization memories (ECM), also known as conductive-bridge memories (CBRAM) or programmable metallization cells (PMCs) [2,3]. In these systems, cation redox reactions lead to the growth and dissolution of nanoscale metal dendrites that reversibly short-circuit the electrodes to create two memory states with very different resistances[2,3].

In contrast to solid-state devices, resistive switching in liquids has received much less attention, in spite of its potential to open new and different applications, e.g. for control, logic, or memory in microfluidic devices. Indeed, liquid-state ECM would appear to be impossible because rapid electrodeposition in bulk liquids becomes unstable to the growth of fragile, fractal deposits, which cannot be grown and dissolved reversibly[6-8]. To our knowledge, the only previous attempt at resistive switching in an aqueous system involved adding a drop of water in a gap between microelectrodes to induce the local formation and 
dissolution of $\mathrm{Ag}$ dendrites[9], albeit without demonstrating the long-term stability of ON/OFF cycles.

In this paper, we report stable, reversible resistive switching in an aqueous system. The required control of metal electrodeposition[10,11] is achieved by exploiting the new physics of over-limiting current (faster than diffusion)[10,12-14] and deionization shock waves in charged nanochannels[10,15-18] and porous media[11,14,16,19-21]. In bulk liquid electrolytes, the morphological instability leading to dendritic growth results from diffusion limitation[22], but when the electrolyte is confined within negatively charged nanopores, surface transport of cations through the electric double layers can sustain over-limiting current and lead to the formation of stable deionization shock waves (jumps in ion concentrations and the electric field) that propagate against the applied current[12,18,20,21]. Deionization shocks have the general structure of a "diffusive wave", similar to the diffusion layer that precedes dendritic electrodeposition[22-24], but followed instead by a region of strong salt depletion in the nanopores, where large electric fields drive surface conduction and stabilize uniform metal growth[11,16]. In ordered nanopores with unmodified surfaces, metal nanowire growth is difficult to synchronize[25], but our group has showed that shock electrodeposition with charge-modified surfaces offers a powerful new means of controlling rapid metal growth, in both ordered[10] and disordered[11] nanopores. Interestingly, the field of solid-state ReRAM has just come to a similar realization[26], that ionic shock waves (in oxygen vacancies) also control the switching dynamics of memristive elements based on TMO thin films. 


\section{Theory}

The mathematical structure of models in both fields is that of a nonlinear driftdiffusion equation for explaining the fundamental connection between liquid-state and solidstate ion-concentration shock waves and their analogous applications in resistive switching devices.

$$
\frac{\partial c}{\partial t}+v(c) \frac{\partial c}{\partial x}=D \frac{\partial^{2} c}{\partial x^{2}}
$$

In simple models of solid-state ReRAM, the local resistivity along a conducting path is proportional the oxygen vacancy concentration $(\sim c)$, and thus so are the local electric field $(E=I)$ and vacancy drift velocity $(v=E)$ at constant applied current $(I)$. In that case $(v(c) \sim c)$, the Nernst-Planck equation (1) reduces to Burgers equation, the simplest model of shock propagation by forward wave breaking ( $v v>0)$, which captures the essential nonlinearities of gas dynamics, water waves, and glaciers[27]. Physically, oxygen vacancies experience larger electric fields and drift faster in regions of higher concentration, so the crest of a concentration wave will overtake the trough until a steep gradient is stabilized by diffusion and propagates as a "shock" in the direction of the current.

In neutral binary electrolytes, concentration relaxation occurs by ambipolar diffusion without drift, but in charged electrolytes confined to micro/nanochannels[11,15,28] or "leaky membranes"[16,20,29], the oppositely charged internal surfaces act as dopants in semiconductors or transition metal sites in TMOs, leading to nonlinear drift by 
electromigration and electro-osmotic flow. The relative importance of surface-driven drift compared to bulk ambipolar diffusion grows as the electric field is amplified by decreasing salt concentration and enables over-limiting current, faster than diffusion[11,12,14]. For negatively charged surfaces, the effective drift velocity scales as $v(c) \sim{ }_{s} I /\left(c_{s}\right)$, where ${ }_{s}<0$ is the surface charge per volume, since excess cations in the double layers of concentration ${ }_{s}$ drift in the local electric field $(E=I)$, which scales with the total resistivity $\left(\sim\left(\begin{array}{ll}c & \end{array}\right)^{1}\right)[16,20]$. In this case, as in traffic flow[30], concentration waves break backwards, since crests move slower than troughs $(v v<0)$. Physically, regions of salt depletion have greater resistivity and amplified electric fields, which drive further depletion by electromigration through the double layers. Electro-osmotic flows further enhance salt depletion via electro-hydrodynamic dispersion, which has thus far resisted a simple description by homogenized equations such as Eq. (1) [12-14,18,28,29].

Shock electrodeposition occurs when electrodeposition is preceded and regulated by a deionization shock wave. Since modeling is quite challenging with diffuse charge, electro-convection and moving boundaries, shock electrodeposition has only been studied experimentally to date[10,11]. In anodic aluminum oxide (AAO) with parallel $300 \mathrm{~nm}$ pores and negatively charged surface coatings, shock electrodeposits grow uniformly along the surfaces at high currents, fed by surface conduction of cations through thin double layers[10]. In membrane materials with smaller, randomly intersecting pores, such as cellulose nitrate (200-300nm) and polyethylene (50nm), shock electrodeposition leads to macroscopically flat deposits, which can be reversibly cycled through the material[11]. 
Fig. 1. Cross-sectional sketch of deionization shocks forming ahead of metal electrodeposition by surface conduction in charged nanopores at high rates, as demonstrated in Ref. [10] for 300nm AAO nanopores with thin double layers and applied here to resistive switching in smaller 50nm PC nanopores with thick double layers in the ion depletion zone. This schematic depicts only one nanopore in the PC membrane.

The observed stability of electrodeposition/dissolution cycles in these cases may be attributable to double layer overlap in the depleted region behind the shock wave, which leads to more uniform excess conductivity and thus more stable shock propagation, according to theory[16]. In order to observe deionization shocks, however, the surface charge must be small compared to the initial bulk salt concentration[20], corresponding to thin double layers. Therefore, we predict that the ideal nanopore radius for programmable metallization by shock electrodeposition should lie in between the Debye screening lengths of the concentrated and depleted solutions, e.g. $10-300 \mathrm{~nm}$ for $1 \mathrm{mM}-1 \mu \mathrm{M}$, so that the double layers are thin ahead of the shock and thick behind it, as sketched in Fig. 1.

\section{Experimental}

Our prototype for aqueous resistive switching utilizes polycarbonate (PC) membranes with ordered nano-sized cylindrical parallel pores $(50 \mathrm{~nm}$ in diameter, $6 \mu \mathrm{m}$ in length) whose surface charge is modified by layer-by-layer deposition of polyelectrolyte layers of alternating charge. Polydiallyldimethylammonium chloride (pDADMAC) is directly deposited on a bare $\mathrm{PC}$ membrane to make $\mathrm{PC}(+)$ with positive surface charge. A negatively charged PC(-) membrane is then obtained by coating negative polyelectrolytes (poly(styrenesulfonate), pSS) on the pDADMAC-coated $\mathrm{PC}(+)$ membrane. The PC 


\section{Results and discussion}

The current-voltage curves (Fig. 2a) show the expected dependence on the surface charge of the porous medium during over-limiting current with metal 
Fig. 2. (a) Linear sweep voltammetry (LSV) of $\mathrm{PC}(+)$ and $\mathrm{PC}(-)$ membranes of exposed area $1 \mathrm{~cm}^{2}$ between $\mathrm{Cu}$ disk electrode and $\mathrm{Au}$ wafer electrode in $1 \mathrm{mM} \mathrm{CuSO}_{4}$ at $2 \mathrm{mV} / \mathrm{s}$. The outer solution is water. The potential of the $\mathrm{Au} / \mathrm{Si}$ working electrode is measured relative to the $\mathrm{Cu}$ counter-electrode, so that $\mathrm{Cu}$ electrodeposition on the working electrode (negative reduction current) occurs at negative voltages. (b) SEM image of a transverse cross section of a PC membrane, showing randomly dispersed, non-intersecting pores of $50 \mathrm{~nm}$ mean diameter.

The morphologies of electrodeposits forming the short circuits were not characterized in this study, since it is difficult to isolate the relatively small number of nanopores filled with copper, but based on our previous imaging experiments on copper electrodeposits in anodized aluminum oxide (AAO) membranes with similar surface charge modifications[10], it is likely that the short circuit structures consist of mostly space-filling copper nanorods, as well as some copper nanotubes coating the surfaces, depending on the variable local current density within each nanopore[25]. On the other hand, positive surface charge suppresses metal growth as a result of oppositely-directed surface conduction, and the 
copper deposits grown in $\mathrm{PC}(+)$ are typically very short and never approach the $\mathrm{Cu}$ counterelectrode, as in our previous work[10].

Fig. 3. LSV of $\mathrm{PC}(+)$ and $\mathrm{PC}(-)$ membranes of exposed area $1 \mathrm{~cm}^{2}$ between $\mathrm{Cu}$ disk electrode and $\mathrm{Au}$ wafer electrode in distilled water at $2 \mathrm{mV} / \mathrm{s}$. The outer solution is also distilled water.

The current-voltage relations for $\mathrm{PC}(+)$ and $\mathrm{PC}(-)$ were also examined with distilled water as the inner solution (Fig. 3). The current of $\mathrm{PC}(-)$ is much higher than that of $\mathrm{PC}(+)$, which provides evidence that growth of copper nanowires is driven by surface conduction of $\mathrm{Cu}^{2+}$ ions originating from dissolution of the $\mathrm{Cu}$ anode. PC(-) showed an increase in current driven by surface conduction regardless of the type of inner solution. The difference is that PC(-) showed a short circuit with $1 \mathrm{mM} \mathrm{CuSO}_{4}$ as the inner solution, while, in distilled water, PC(-) showed only gradual increase in current without a short circuit. This indicates that the very low concentration of $\mathrm{Cu}^{2+}$ ions resulting from dissolution of the $\mathrm{Cu}$ anode is not sufficient to grow copper nanowires all the way to a short circuit when distilled water is used as inner solution.

Fig. 4. Resistive switching of by copper electrodeposition (brown) in the PC(-) membrane in $1 \mathrm{mM} \mathrm{CuSO}_{4}$ at $1 \mathrm{mV} / \mathrm{s}$ with sketches of the stages of metallization within the charged nanopores.

After getting the first short circuit of PC(-) membrane from the LSV (Fig. 2a), an oxidation potential $(0.8 \mathrm{~V})$ is applied at the $\mathrm{Au}$ electrode to detach $\mathrm{Cu}$ nanowires from the $\mathrm{Cu}$ electrode until the current is decreased to less than $0.05 \mathrm{~mA}$. Repeated cycles of resistive 
Fig. 5. Resistive switching of copper in the $\mathrm{AAO}(-)$ membrane of exposed area $1 \mathrm{~cm}^{2}$ between $\mathrm{Cu}$ disk electrode and $\mathrm{Au}$ wafer electrode in $10 \mathrm{mM} \mathrm{CuSO}_{4}$ at $1 \mathrm{mV} / \mathrm{s}$. The outer solution is water.

In order to test the effect of nanopore radius, we also studied resistive switching AAO(-) membranes with $300 \mathrm{~nm}$ pores from our previous work[10], whose diameter is comparable to the Debye length in deionized water. In order to further reduce double layer overlap, we also used a larger initial salt concentration of $10 \mathrm{mM}$, which increases the depleted concentration at the same current[14,21]. Under these conditions, similar behavior of resistive switching was observed in $\mathrm{AAO}(-)$ as in $\mathrm{PC}(-)$, but the cycling capability is 
Fig. 6. (a) Cycling test at negative pulses (ON state) of $-0.1 \mathrm{~V}$ for $20 \mathrm{~s}$ followed by positive pulses (OFF state) of $0.4 \mathrm{~V}$ for $30 \mathrm{~s}$. (b) Long-term ON/OFF cycling over 7 hours. Copper is electrodeposited and electrostripped in $\mathrm{PC}(-)$ in an inner solution of $<1 \mathrm{mM} \mathrm{CuSO}_{4}$ and distilled water as the outer solution.

Cycling tests were performed with the most stable PC(-) membrane by applying repeated pulses of negative and positive voltages (Fig. 6). The ON state current of $-0.1 \mathrm{~mA}$ was found to be very reproducible and stable (Fig. 6a). A consistent retention time on the order of seconds $(\sim 7 \mathrm{~s})$ is required to reach the OFF state after applying positive voltage. Long-term cycling between ON/OFF states for over 7 hours shows fairly good stability and reproducibility under the same experimental conditions (Fig. 6b). Although the ON state current is gradually decreasing, the OFF state current remains almost constant, and robust resistive switching is observed throughout the experiments. Even after 500 cycles, the current still reliably jumps by two orders of magnitude between the ON and OFF states with a consistent response time $<10 \mathrm{~s}$.

The slowly decaying ON state current may be attributable to the gradual exchange of ions between the inner and outer solutions, which could be controlled in future designs in order to extend the cycle life. We have already noted that a more concentrated outer 
solution tends to cause excess metallization as additional cations diffuse into the PC(-) nanopores, which leads to larger ON currents but also difficulty switching back to the OFF state,. In the present prototype with distilled water as the outer solution, cations slowly leak out from the nanopores, and the initial concentration of the inner solution $(1 \mathrm{mM})$ becomes significantly reduced. As a result, the number of $\mathrm{Cu}$ nanowires in metallic contact with the $\mathrm{Cu}$ disk electrode in the $\mathrm{ON}$ state is decreased, leading to higher resistance and lower current. Nevertheless, we can still easily tell the difference between ON and OFF state currents after 500 cycles over 7 hours. The stability and reliability of ON/OFF switching could be improved by better sealing the negatively charged nanopores against the electrodes, surrounding them with positively charged polymer layers, adjusting the concentration of the outer solution, or other methods of controlling to cation exchange with the inner solution.

\section{Conclusions}

Robust resistive switching in an aqueous porous medium by copper metalization in negatively charged nanopores was demonstrated. The ECM-type switching process exploits the stability and control of metal electrodeposition afforded by deionization shock waves, which form during the passage of over-limiting current by electromigration through the electric double layers of the nanopores. After some optimization of the geometry, materials and protocols, it should be possible to develop individually accessible nanopores for aqueous ReRAM with subsecond response times, lasting for thousands of ON/OFF cycles. Applications of aqueous resistive switching include control in lab-on-a-chip devices for micro-total analysis of chemical samples, biomedical diaognostics or drug delivery, and 
related logic and memory.

\section{Acknowledgements}

This work was supported in part by a grant from the MIT Energy Initiative. MZB acknowledges partial support from the Global Climate and Energy Project at Stanford University and by the US Department of Energy, Basic Energy Sciences through the SUNCAT Center for Interface Science and Catalysis.

\section{References}

[1] L. O. Chua, S. M. Kang, Memristive devices and systems, Proc. IEEE, 64 (1976) 209.

[2] I. Valov, R. Waser, J. R. Jameson, M. N. Kozicki, Electrochemical metallization memories-fundamentals, applications, prospects, Nanotechnology, 22 (2011) 254003.

[3] R. Waser, R. Dittmann, G. Staikov, K. Szot, Redox-based resistive switching memories - nanoionic mechanisms, prospects, and challenges, Adv. Mater., 21 (2009) 2632.

[4] R. Waser, M. Aono, Nanoionics-based resistive switching memories., Nat. Mater., 6 (2007) 833.

[5] D. B. Strukov, G. S. Snider, D. R. Stewart, R. S. Williams, The missing memristor found, Nature, 453 (2008) 80.

[6] R. M. Brady, R. C. Ball, Fractal growth of copper electrodeposits, Nature, 309 (1984) 225.

[7] M. Rosso, Electrodeposition from a binary electrolyte: new developments and applications, Electrochim. Acta, 53 (2007) 250.

[8] V. Fleury, J. N. Chazalviel, M. Rosso, Theory and experimental evidence of electroconvection around electrochemical deposits, Phys. Rev. Lett., 68 (1992) 2492.

[9] X. Guo, C. Schindler, S. Menzel, R. Waser, Understanding the switching-off mechanism in Ag+ migration based resistively switching model systems, Appl. Phys. Lett., 91 (2007) 133513.

[10] J.-H. Han, E. Khoo, P. Bai, M. Z. Bazant, Over-limiting current and control of dendritic growth by surface conduction in nanopores, Sci. Rep., 4 (2014) 7056.

[11] J.-H. Han, M. Wang, P. Bai, F. R. Brushett, M. Z. Bazant, Dendrite suppression by shock electrodeposition in charged porous media, Sci. Rep., 6 (2016) 28054.

[12] E. V. Dydek, B. Zaltzman, I. Rubinstein, D. S. Deng, A. Mani, M. Z. Bazant, Overlimiting current in a microchannel, Phys Rev Lett., 107 (2011) 118301.

[13] A. Yaroshchuk, E. Zholkovskiy, S. Pogodin, V. Baulin, Coupled concentration 
polarization and electroosmotic circulation near micro/nanointerfaces: Taylor-Aris model of hydrodynamic dispersion and limits of its applicability, Langmuir, 27 (2011) 11710

[14] D. Deng, E. V. Dydek, J.-H. Han, S. Schlumpberger, A. Mani, B. Zaltzman, M. Z. Bazant, Overlimiting current and shock electrodialysis in porous media, Langmuir, 29 (2013) 16167.

[15] A. Mani, T. Zangle, J. Santiago, On the propagation of concentration polarization from microchannel-nanochannel interfaces Part I: analytical model and characteristic analysis, Langmuir, 25 (2009) 3898.

[16] A. Mani, M. Z. Bazant, Deionization shocks in microstructures, Phys. Rev. E 84 (2011) 061504.

[17] T. Zangle, A. Mani, J. Santiago, On the propagation of concentration polarization from microchannel-nanochannel interfaces Part II: numerical and experimental study, Langmuir, 25 (2009) 3909.

[18] S. Nam, I. Cho, J. Heo, G. Lim, M. Z. Bazant, D. J. Moon, G. Y. Sung, S. J. Kim, Experimental verification of overlimiting current by surface conduction and electroosmotic flow in microchannels, Phys. Rev. Lett., 114 (2015) 114501.

[19] A. Yaroshchuk, Over-limiting currents and deionization "shocks" in current-induced polarization: Local-equilibrium analysis, Adv. Colloid Interface Sci., 183-184 (2012) 68.

[20] E. V. Dydek, M. Z. Bazant, Nonlinear dynamics of ion concentration polarization in porous media: The leaky membrane model, AIChE Journal, 59 (2013) 3539.

[21] S. Schlumpberger, N. B. Lu, M. E. Suss, M. Z. Bazant, Scalable and continuous water deionization by shock electrodialysis, Environ. Sci. Technol. Lett. , 2 (2015) 367.

[22] J. Elezgaray, C. Léger, F. Argoul, Linear stability analysis of unsteady galvanostatic electrodeposition in the two-dimensional diffusion-limited regime, J. Electrochem. Soc., 145 (1998) 2016.

[23] M. Z. Bazant, Regulation of ramified electrochemical growth by a diffusive wave, Phys. Rev. E, 52 (1995) 1903.

[24] D. Barkey, P. Laporte, The dynamic diffusion layer in branched growth of a conductive-polymer aggregate in a 2-D electrolysis cell, J. Electrochem. Soc., 137 (1990) 1655.

[25] S. Shin, T. T. Al-Housseiny, B. S. Kim, H. H. Cho, H. A. Stone, The race of nanowires: morphological instabilities and a control strategy, Nano Lett., 14 (2014) 4395.

[26] S. Tang, F. Tesler, F. G. Marlasca, P. Levy, V. Dobrosavljević, M. Rozenberg, Shock waves and commutation speed of memristors, Phys. Rev. X, 6 (2016) 011028.

[27] G. B. Whitham. Linear and Nonlinear Waves, Wiley-Interscience, New York, 1974.

[28] C. P. Nielsen, H. Bruus, Concentration polarization, surface currents, and bulk advection in a microchannel, Phys. Rev. E, 90 (2014) 043020.

[29] M. Schmuck, M. Z. Bazant, Homogenization of the Poisson-Nernst-Planck equations for ion transport in charged porous media, SIAM J. Appl. Math., 75 (2015) 1369.

[30] R. Haberman. Mathematical Models: Mechanical Vibrations, Population Dynamics, and Traffic Flow, New Jersey: SIAM, 1998.

[31] D. Grujicic, B. Pesic, Electrodeposition of copper: the nucleation mechanisms, Electrochim. Acta, 47 (2002) 2901. 


\section{Graphical Abstract}

Robust resistive switching by copper electrodeposition in an aqueous porous medium was achieved from deionization shock waves generating during the passage of over-limiting current by electromigration through the electric double layers of negatively charged nanopores.

Shock electrodeposition in negatively charged nanopores
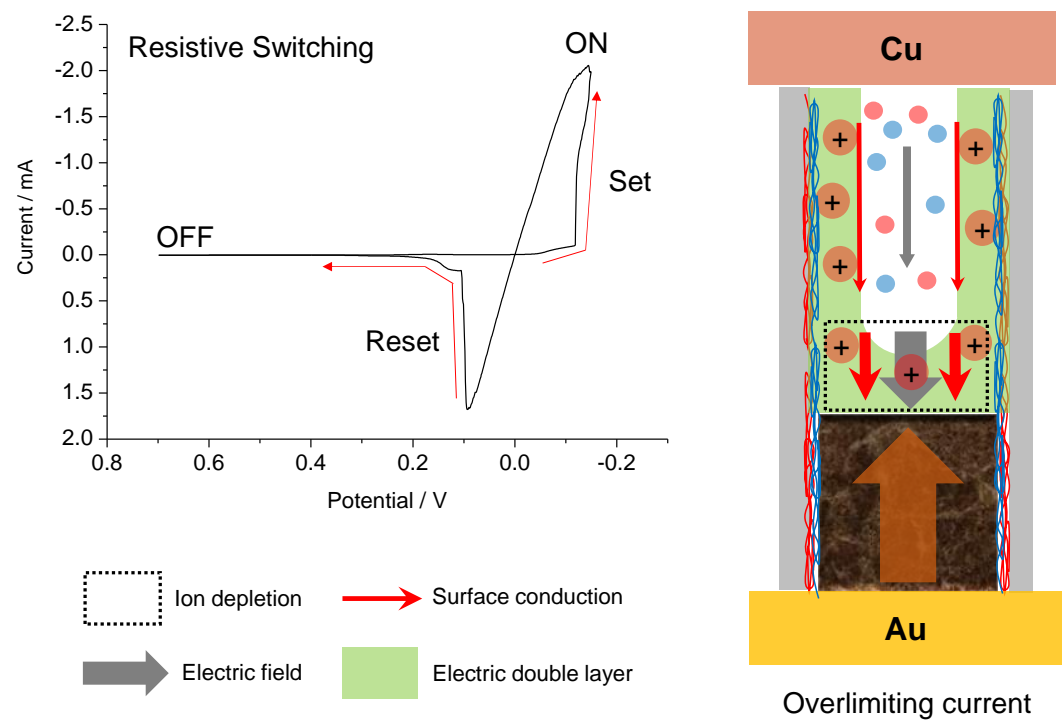

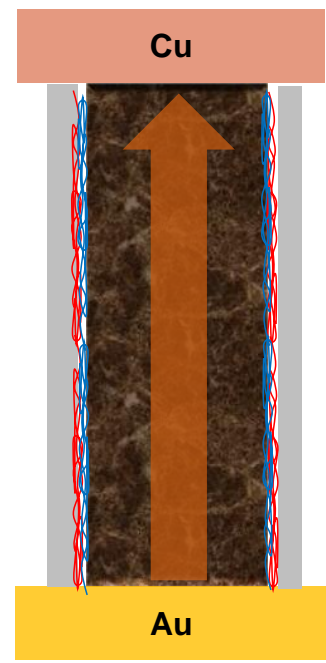

Short circuit 

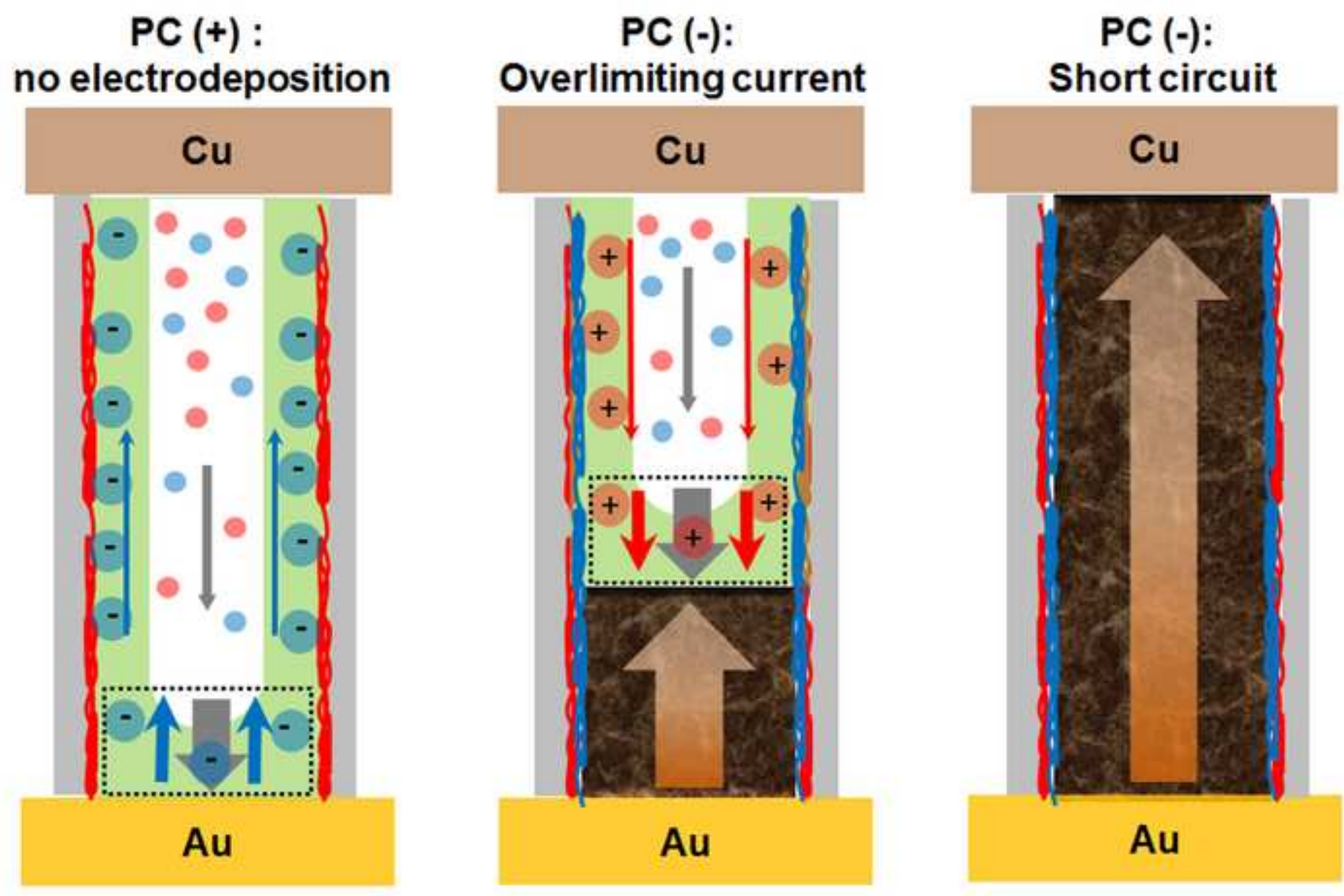

I............. In depletion

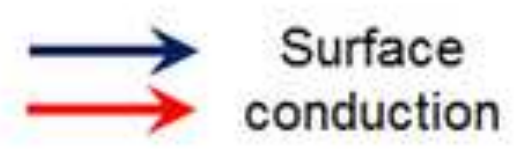

Electric field

Electric double layer 


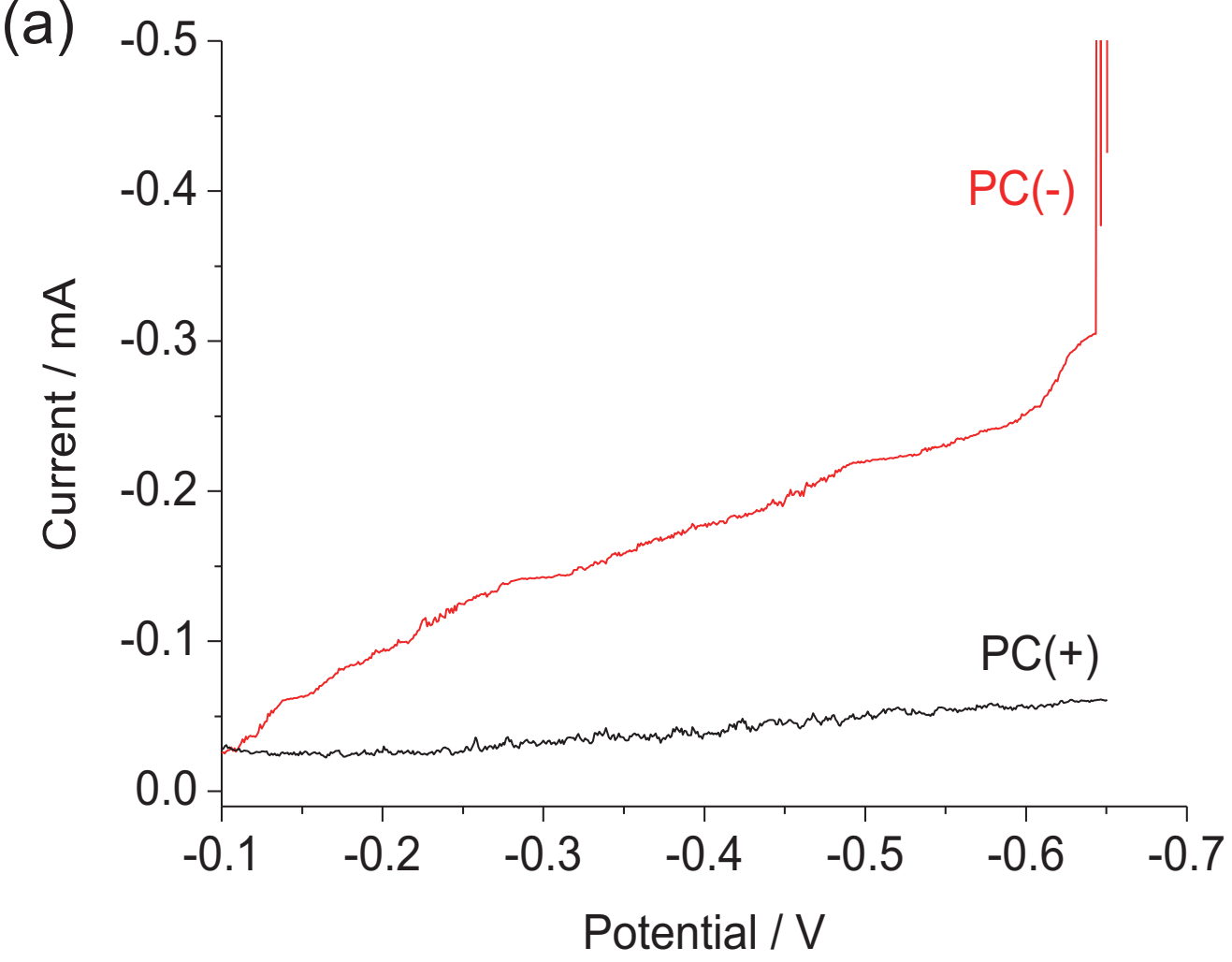

(b)

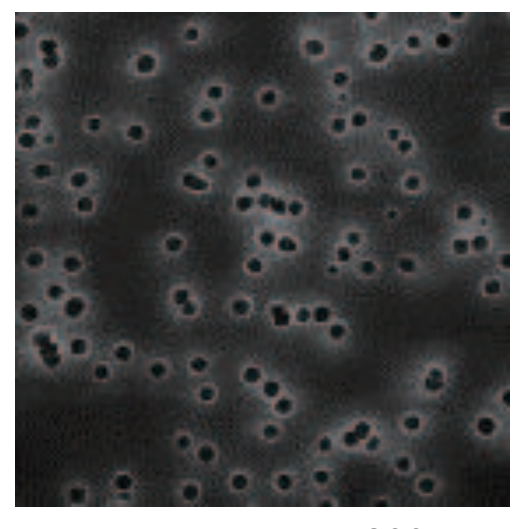

$200 \mathrm{~nm}$

Potential / V 
Figure 3

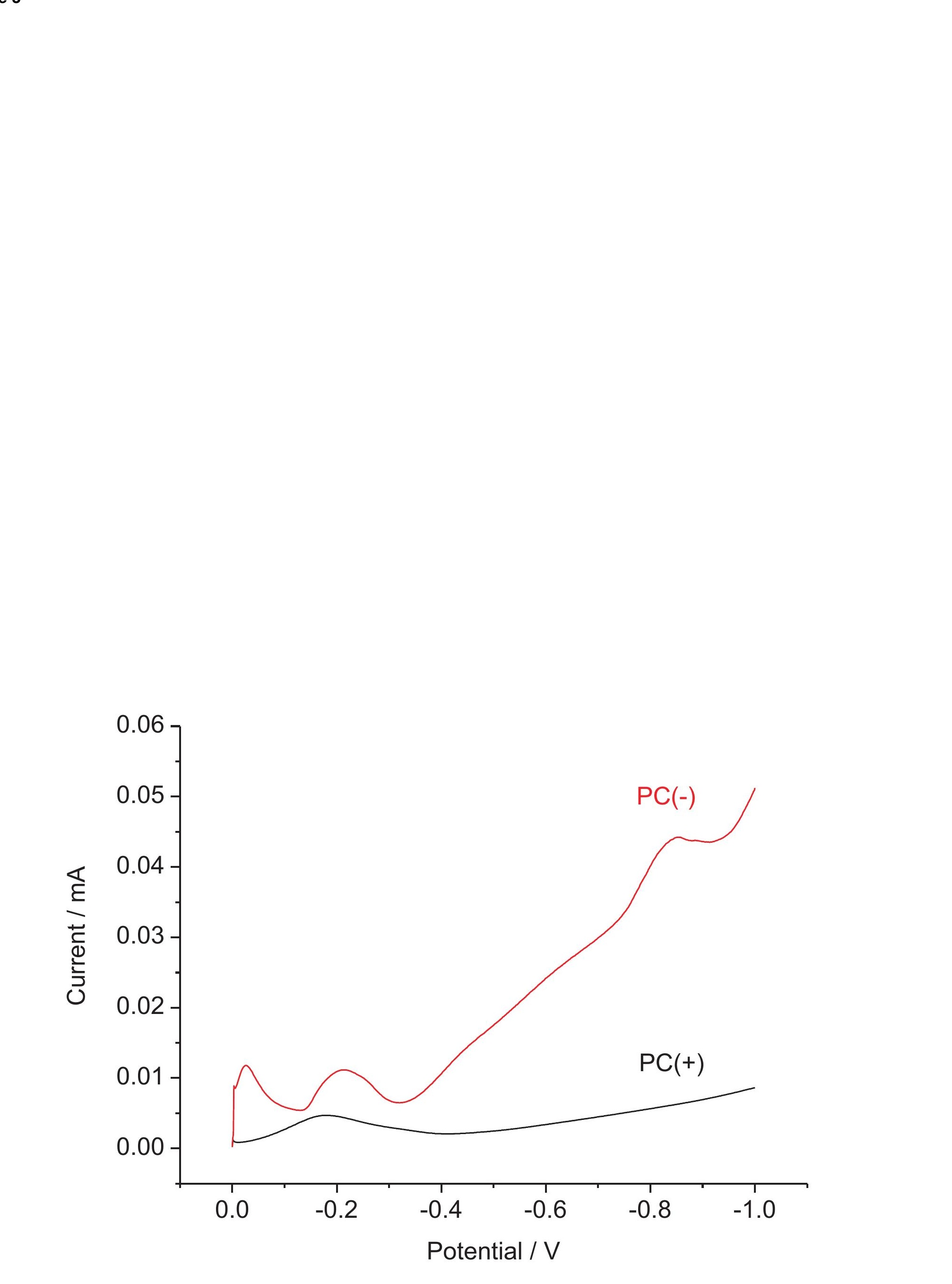

\section{Figure 3}

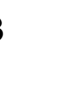




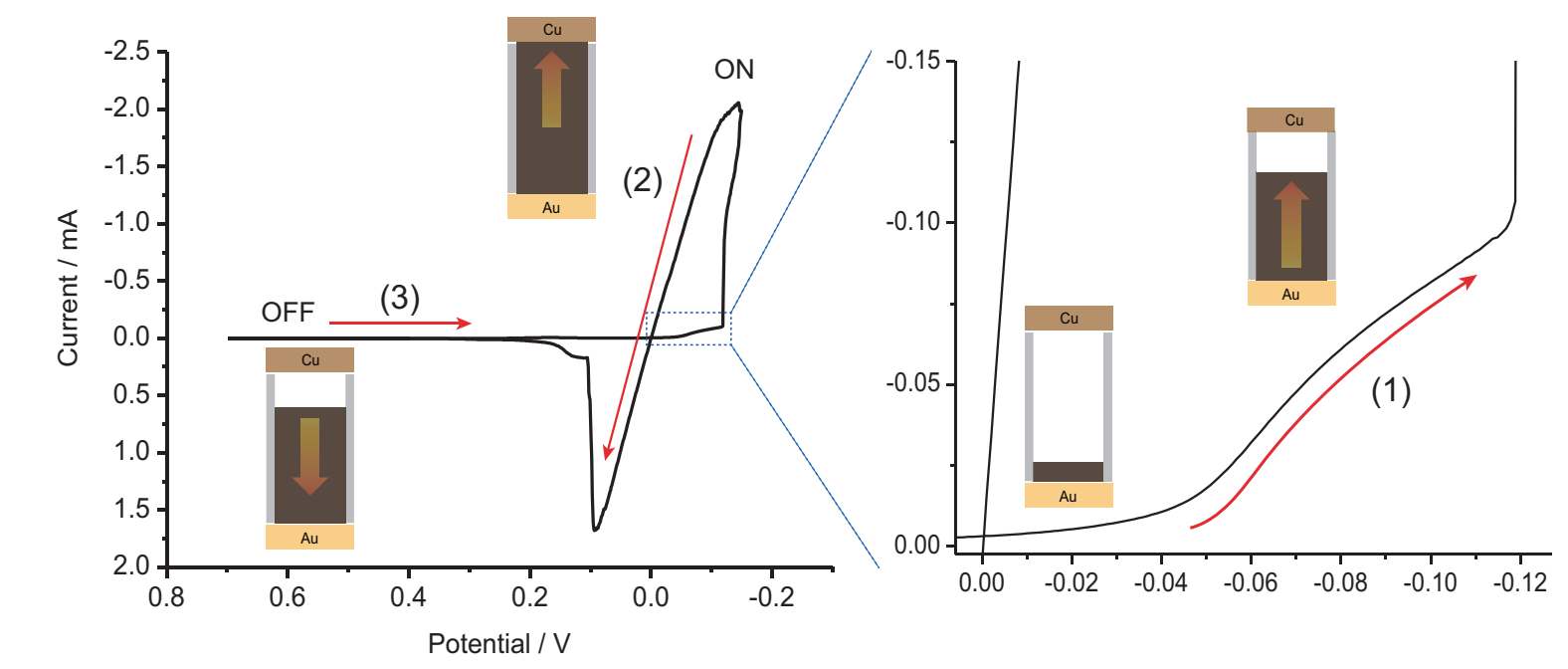

Figure 4 


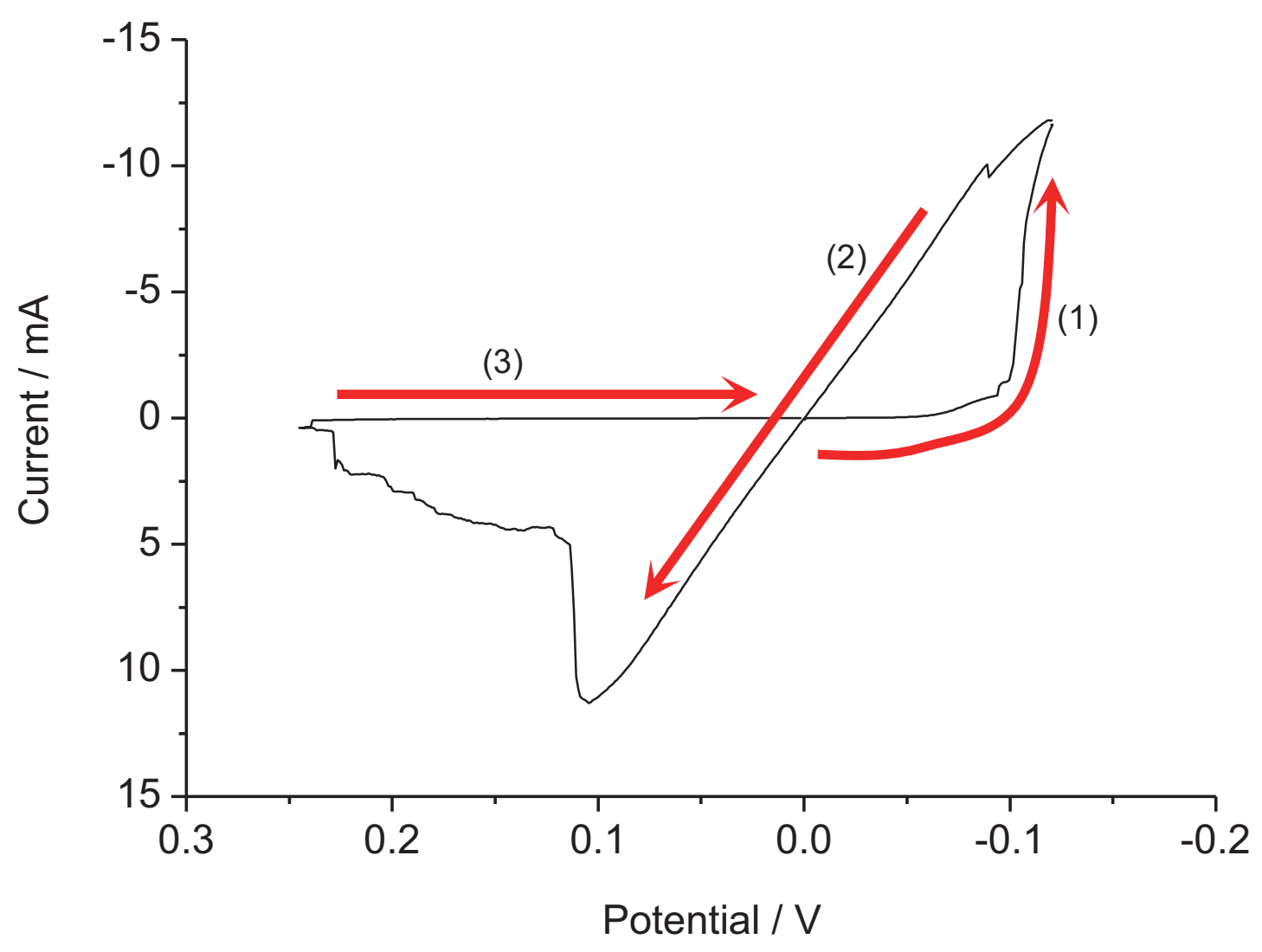

Figure 5$$
\text { - }
$$$$
50
$$ 
Figure 6a

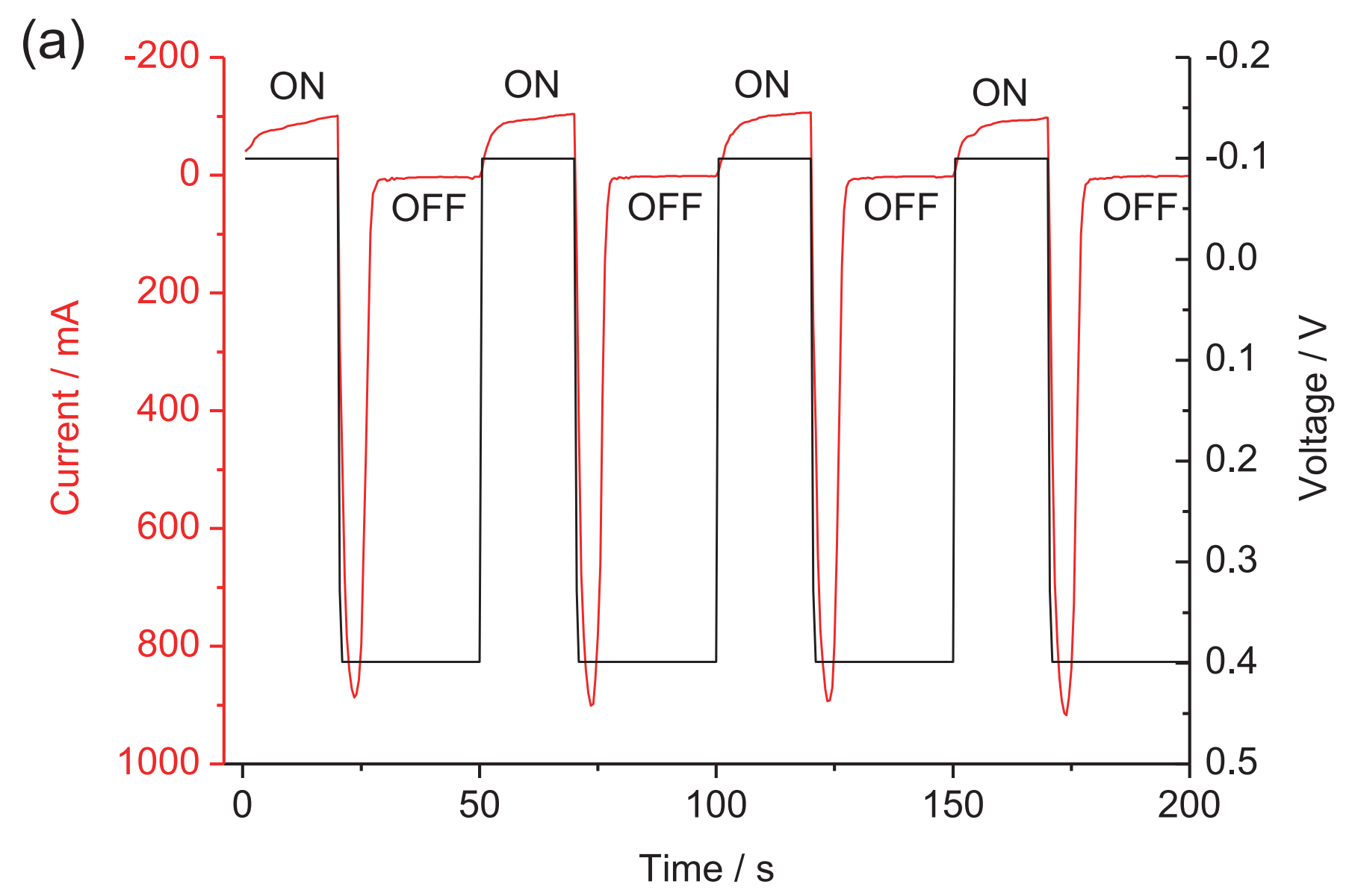


(b) 140

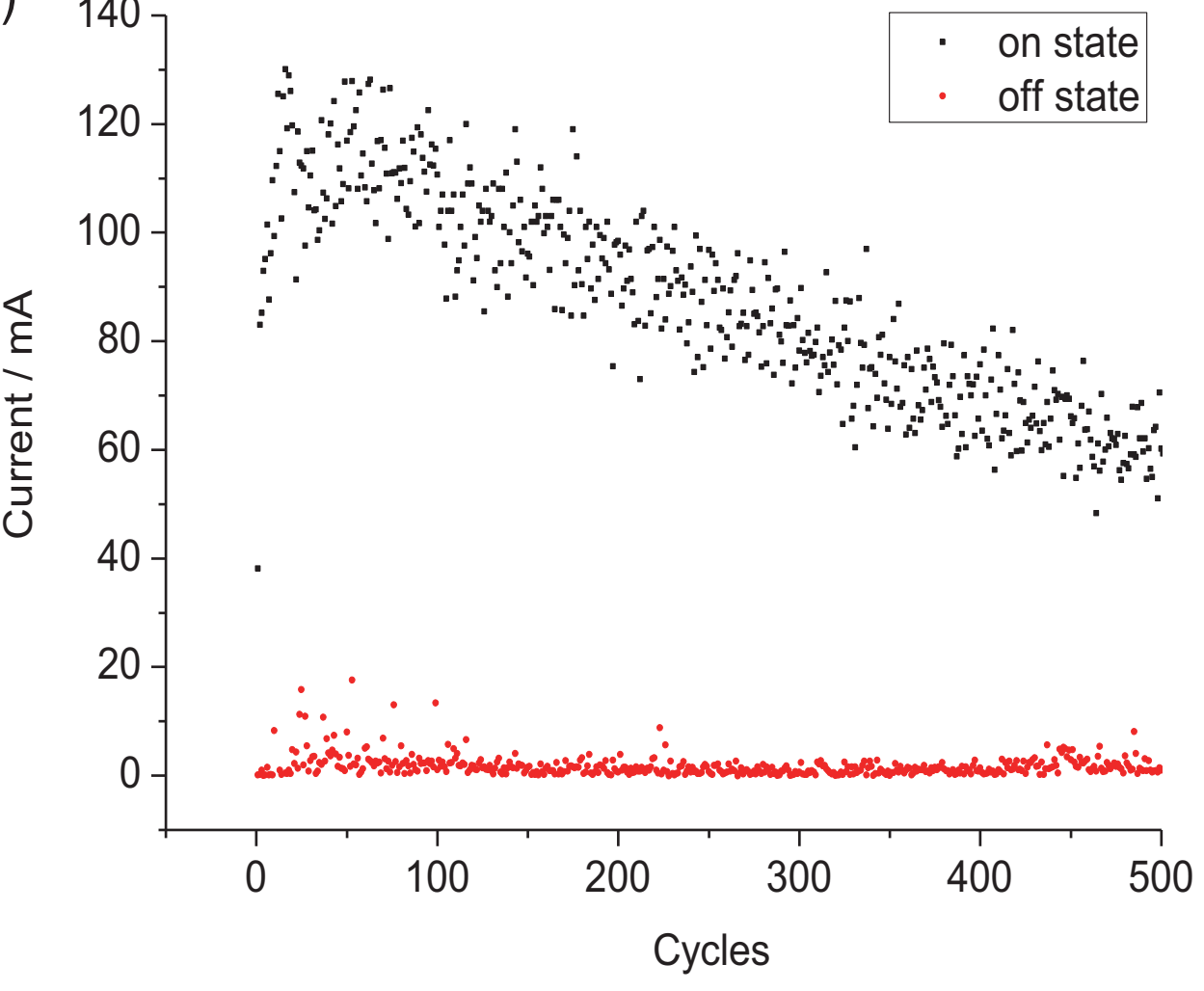

